\title{
28 Resarch Square \\ Clustering of Cancer Data Based on Stiefel Manifold for Multiple Views
}

\section{Jing Tian}

Xinjiang University

Jianping Zhao ( $\nabla$ zhaojianping@126.com )

Xinjiang University

Chun-hou Zheng

Xinjiang University

\section{Research Article}

Keywords: Stiefel manifold, multi-view clustering, cancer data, optimization model, linear search algorithm

Posted Date: February 12th, 2021

DOI: https://doi.org/10.21203/rs.3.rs-154286/v1

License: (c) (1) This work is licensed under a Creative Commons Attribution 4.0 International License. Read Full License 


\title{
Clustering of Cancer Data Based on Stiefel Manifold for Multiple Views
}

\author{
Jing Tian ${ }^{1}$, Jian-ping Zhao ${ }^{1, *}$, Chun-Hou Zheng ${ }^{1,2,1}$
}

${ }^{1}$ College of Mathematics and System Sciences, Xinjiang University, Urumqi, China

${ }^{2}$ School of Computer Science and Technology, Anhui University, Hefei, China

\begin{abstract}
Background: In recent years, various sequencing techniques have been used to collect biomedical omics datasets. It is usually possible to obtain multiple types of omics data from a single patient sample. Clustering of these datasets has proved to be valuable for biological and medical research and helpful to reveal data structures from multiple collections. However, such data often have small sample size and high dimension. It is difficult to find a suitable integration method for structural analysis of multiple datasets.
\end{abstract}

Results: In this paper, a multi-view clustering based on Stiefel manifold method (MCSM) is proposed. Firstly, we established a binary optimization model for the simultaneous clustering problem. Secondly, the optimization problem solved by linear search algorithm based on Stiefel manifold. Finally, we integrated the clustering results obtained from three omics by using k-nearest neighbor method. We applied this approach to four cancer datasets on TCGA. The result shows that our method is superior to several state-of-art methods, which depends on the hypothesis that the underlying omics cluster class is the same.

Conclusion: Particularly, our approach has better performs when the underlying clusters are

${ }^{1}$ Corresponding authors 
inconsistent. For patients with different subtypes, both consistent and differential clusters can be identified at the same time.

Keywords: Stiefel manifold; multi-view clustering; cancer data; optimization model; linear search algorithm

\section{Introduction}

One of the challenges of cancer treatment is how to identify tumor subtypes, which can help to provide patients with specific treatment. Meanwhile, with the continuous development of all kinds of sequencing technologies, a lot of high flux data have been produced (Zheng et al. 2016). For cancer subtypes identification, integration of different types of omics data to unravel the molecular mechanism of complex diseases becomes more and more important (Zhang et al., 2016). On the one hand, multiple omics data of different subtypes of cancer provided more detailed information. On the other hand, it leaded to the new challenge for data analysis. Different levels of multiple omics data often show different types, they have different correlation structure statistical properties and expressions (Zhang et al., 2011). In addition, the same tumor specimens from different levels of data are also unlikely to be independent. Therefore, how to reasonably integrate the multiple omics data to accurately predict cancer subtypes becomes a challenging and interesting research (Bickel et al., 2009).

Recently, many strategies have emerged to integrate multi-omics data for association studies. The main objectives of these studies usually are to understand the inter-relationships among various omics data and to detect phenotype-related modules (Manko et al., 2010; Kumar et al., 2011; Yuan et al., 2011). These Multi-view data integration methods can be divided into three categories: early 
integration, late integration and intermediate integration. Early integration mainly merged all data types into a single dataset and analyzed them directly (Manko et al., 2010). Late integration is the process of analyzing each data type, then combining the results for integration (Fridly et al., 2012). Intermediate integration converts each data type into an intermediate representation, which is then consolidated for analysis (Cancer Genome Atlas Research Network et al., 2012).

In recent years, many new methods are also published for clustering. For example, Similarity Network Fusion (SNF) method (Wang et al., 2014) constructed the similarity network for each data type, and then used the iterative method to fuse them into a similar network. The final clustering is obtained by spectral clustering of fusion networks. Some multi-view clustering methods based on spectral clustering have also been proposed (Huang et al., 2012; Kumar et al., 2011; Zhang et al., 2015). They used different integration methods to combine the spectral clustering results from a single view. The Affinity Aggregation for Spectral Clustering (AASC) algorithm (Huang et al., 2012) introduced weights in the spectral clustering of each view, and then added them together to optimize the weights in the calculation.

However, these methods were put forward based on a basic hypothesis that the underlying omics cluster is the same. In actual situation, there are differences in clusters except for the same cluster (Yuan et al., 2011). In the process of integrated clustering, data clustering was carried out for each view and cluster alignment was carried out for different views, which could handle this situation (Kumar et al., 2011; Zhang et al., 2015; Chen et al., 2017). However, the method (Kumar et al., 2011) tended to obtain the local optimal as described above, and the methods (Zhang et al., 2015; Chen et al., 2017) relax excessively the original multi-view point specific tangent condition, so that the information of each viewpoint may be lost. In the paper (Yu et al., 2019), the authors proposed 
the Multi-View Clustering using Manifold Optimization (MVCMO) method considering the diversity of the cluster. Consistent clusters and different clusters can be identified in each group. This method can effectively identify the cluster of differences, and this theory is also used in our method.

In order to improve the algorithm stability of MVCMO (Yu et al., 2019), we introduce the "Heat Kernel" to measure similarity between patients. And we use Backtracking Line Search to find the optimal solution more accurately. In this study, we propose a Multi-view Clustering based on Stiefel Manifold (MCSM) method for multi-view clustering problems with potential clusters. Firstly, we introduce a "Heat Kernel" to measure similarity. The patient-patient similarity network is constructed using k-nearest neighbor (KNN) method. Then we establish a binary optimization model for the simultaneous clustering problem. The solving process of the objective optimization problem is divided into three steps. First, we project our target function onto each of Stiefel manifold's tangent vector Spaces. Second, we do Backtracking Line Search on Stiefel manifold for the objective problem. Third, we retract the found points to the manifold with singular value decomposition. Finally, the KNN method is used for integrating the obtained clusters from three omics to get the final result. The proposed MCSM method has two highlights. One is that it preserves as much data information of each sample as possible. The other is that it can identify the cluster effectively when the underlying clusters are different. We experiment on simulated datasets to see the algorithm's performance when there are potential clusters. The experimental results on simulated datasets and several multiple omics datasets from TCGA show that our method has better performance than state-of-art methods.

\section{Datasets and Methods}




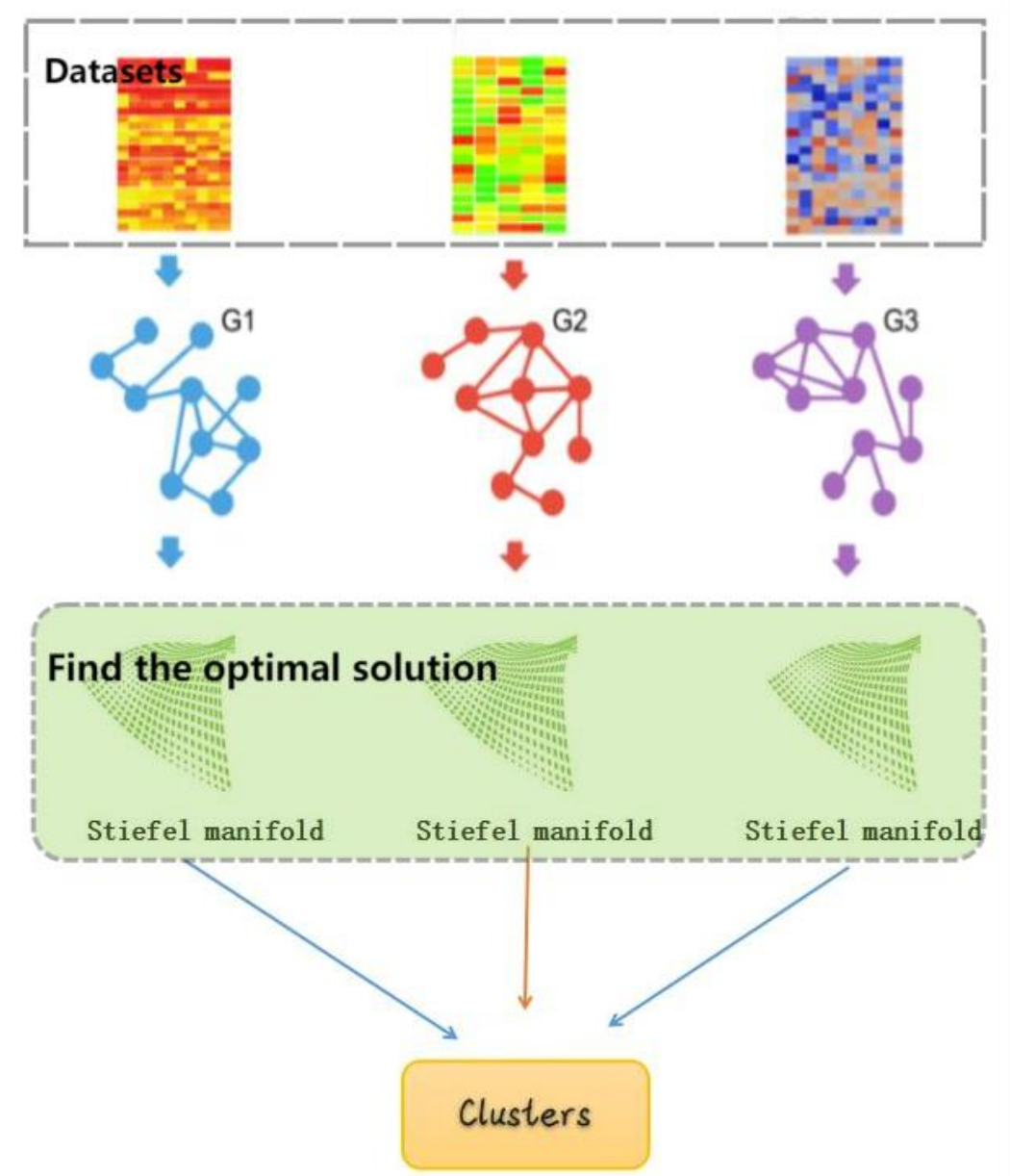

89

Figure 1: The process of MCSM method.

\subsection{Datasets and preprocessing}

In this paper, we selected four cancer datasets in the TCGA for experiment, including gene expression data, miRNA expression data and DNA methylation data from samples of cancer patients.

The cancer datasets include glioblastoma multiforme (GBM) with 215 samples, breast invasive carcinoma (BIC) with 105 samples, Skim Cutaneous Melanoma (SKCM) with 439 samples and Acute Myeloid Leukemia (AML) with 96 samples.

Firstly, if the data of a patient loses more than $20 \%$ in any data type, the patient will be deleted. 
Secondly, if the missing value of a feature in all patients exceeds $20 \%$, it will be filtered out. Thirdly, the K-nearest-neighbor method is adopted to fill in missing data.

Fourthly, we log transform the data set to make it more stable. Finally, each feature is normalized in the constructed network to make it have a standard normal distribution. We performed the following normalization for each data type:

$$
\hat{f}=\frac{f-E(f)}{\sqrt{\operatorname{Var}(f)}} .
$$

where $f$ is the characteristic of sample data, $\widehat{f}$ is the corresponding characteristic after $f$ normalization of $f, \mathrm{E}(f)$ and $\operatorname{Var}(f)$ represent the sample mean and sample variance respectively.

\subsection{Construction of the patient-to-patient similarity graph}

Denoted $\left\{\mathrm{X}^{\mathrm{m}}\right\}_{\mathrm{m}=1}^{\mathrm{M}}$ as multi-view data from $\mathrm{N}$ patient samples, which has $\mathrm{m}$ data type in total. Each $\mathrm{X}^{\mathrm{m}}$ is a matrix of $\mathrm{p}_{\mathrm{m}} \times \mathrm{N}$, then a similar network graph $\mathrm{G}^{\mathrm{m}}$ is constructed to reflect the neighborhood relationship between the samples.

In the similar network of type $\mathrm{m}, \mathrm{G}^{\mathrm{m}}=\left(\mathrm{V}^{\mathrm{m}}, \mathrm{E}^{\mathrm{m}}, \mathrm{W}^{\mathrm{m}}\right), \mathrm{V}^{\mathrm{m}}$ is vertex set, $\mathrm{E}^{\mathrm{m}}$ is edge set, and $\mathrm{W}^{\mathrm{m}}$ is adjacency matrix. The adjacency matrix of $\mathrm{W}^{\mathrm{m}}$ in graph $\mathrm{G}^{\mathrm{m}}$ is a symmetric matrix.

In this paper, "Heat Kernel" is used to measure the similarity between samples (Ding et al., 2018).

$$
S_{i j}^{m}=\exp \left(-\frac{\left\|x_{i}^{m}-x_{j}^{m}\right\|^{2}}{2 t^{2}}\right), i=1 \cdots, N, j=1 \cdots, N
$$

Next, we construct the K-nearest neighbor graph based on the similarity matrix $S^{m}$. If the vertex has an edge between $v_{i}$ and $v_{j}$, then $W_{i j}^{m}$ represents the edge weight, otherwise 0 .

$$
W_{i j}^{m}=\left\{\begin{array}{l}
S_{i j}^{m}, \quad v_{j} \in N_{i}, \\
0, \text { otherwise. }
\end{array}\right.
$$


here, $\mathrm{N}_{\mathrm{i}}$ is the neighborhood of $\mathrm{v}_{\mathrm{i}}$ (including $\mathrm{v}_{\mathrm{i}}$ ), $\mathrm{N}_{\mathrm{i}}$ with size $\mathrm{k}$, and the number of $\mathrm{k}$ usually depends on the size of the sample. Essentially, we assume that local similarity is more reliable than remote similarity. This is a modest assumption, and it is widely used by other manifold learning algorithms (Ding et al., 2018).

\subsection{Construction of objective optimize problem}

The objective optimize problem of the spectral clustering method is:

$$
\min _{U_{m} \in R^{N \times k}} \operatorname{trace}\left(U_{m}^{T} L_{m} U_{m}\right)
$$

$$
\text { s.t. } U_{m}^{\mathrm{T}} U_{m}=I_{K} \text {. }
$$

Here, the $L_{m}=\left(D_{m}-A_{m}\right)$. The $A_{m}$ is the corresponding adjacency matrix of similar network $\mathrm{G}^{\mathrm{m}}$, and $D_{m}$ is the diagonal matrix constructed using the degree of all the nodes in the $\mathrm{m}$-th network.

Then, used $U_{m}$ for K-means clustering and find its minimum k eigenvectors in order to obtain the clustering labels.

Based on the spectral clustering, (Zhang et al., 2015) proposed a multi-view network clustering method. Its objective optimize problem is:

$$
\begin{gathered}
\min \sum_{\mathrm{m}=1}^{\mathrm{M}} \sum_{\mathrm{k}=1}^{\mathrm{K}} \frac{\left(\mathrm{S}_{, \mathrm{k}}^{\mathrm{m}}\right)^{\mathrm{T}}\left(\mathrm{D}_{\mathrm{m}}-\mathrm{A}_{\mathrm{m}}\right)\left(\mathrm{S}_{, \mathrm{k}}^{\mathrm{m}}\right)}{\left(\mathrm{S}_{, \mathrm{k}}^{\mathrm{m}}\right)^{\mathrm{T}}\left(\mathrm{S}_{, \mathrm{k}}^{\mathrm{m}}\right)}-\beta \sum_{\mathrm{l} \neq \mathrm{m}} \sum_{\mathrm{k}=1}^{\mathrm{K}} \frac{\left(\mathrm{S}_{, \mathrm{k}}^{\mathrm{m}}\right)^{\mathrm{T}}\left(\mathrm{S}_{, \mathrm{k}}^{\mathrm{l}}\right)}{\left\|\mathrm{S}_{, \mathrm{k}}^{\mathrm{m}}\right\|_{2}\left\|\mathrm{~S}_{, \mathrm{k}}^{\mathrm{l}}\right\|_{2}} \\
\text { s.t. } \mathrm{S}_{, \mathrm{k}}^{\mathrm{m}} \in\{0,1\}, \mathrm{i}=1 \cdots, \mathrm{N} ; \mathrm{m}=1 \cdots, \mathrm{M} ; \mathrm{k}=1 \cdots, \mathrm{k} ; \\
\sum_{\mathrm{k}=1}^{\mathrm{K}} \mathrm{S}_{\mathrm{i}, \mathrm{k}}^{\mathrm{m}}=1, \text { for } \mathrm{m}=1 \cdots, \mathrm{M} .
\end{gathered}
$$
of multi-view spectral clustering can be constructed as follows: 
$138 \quad$ where, $\mathrm{L}=\left(\begin{array}{ccc}\mathrm{L}_{1} & \cdots & 0 \\ \vdots & \ddots & \vdots \\ 0 & \cdots & \mathrm{L}_{\mathrm{m}}\end{array}\right)-\beta\left(\begin{array}{ccc}0 & \cdots & \mathrm{I}_{\mathrm{n}} \\ \vdots & \ddots & \vdots \\ \mathrm{I}_{\mathrm{n}} & \cdots & 0\end{array}\right), \mathrm{U}=\left(\begin{array}{c}\mathrm{U}_{1} \\ \mathrm{U}_{2} \\ \vdots \\ \mathrm{U}_{3}\end{array}\right)$.

$139 \quad \beta$ is used to balance the weight parameters between the network and within the network. If we

140 have abundant prior knowledge, we can set it according to prior information. Otherwise, when

141 building a network, we can try to establish a connection at the same level (e.g. similar connection

142 densities) and set it directly to 1 . In our experiment, we set $\beta=1$ directly.

143 However, the optimization problem (6) combines the information of all networks together and

144 will loss the information in each network. The proposed MVSM method still follows the original

145 objective function of multi-view spectral clustering and the construction of Laplace matrix (Yu et 146 al., 2019).

147 The objective optimization problems to be solved are as follows:

$$
\text { s.t. } U_{m}{ }^{T} U_{m}=I
$$

150 When we set $U_{m}=\frac{S^{m}}{\left\|S^{m}\right\|_{2}}$, the objective function $U_{m}^{T} U_{m}=I_{K}$ substitude as $\sum s_{i, j}^{N}=1$. It 151 transforms the constraints for each network into one equation.

\subsection{The solution of objective optimize problem}


Spaces.

$$
\mathrm{TM}_{\mathrm{m}}=\left\{\mathrm{U}_{\mathrm{m}} \mathrm{B}+\left(\mathrm{I}-\mathrm{U}_{\mathrm{m}} \mathrm{U}_{\mathrm{m}}^{\mathrm{T}}\right) \mathrm{C}: \mathrm{B}=-\mathrm{B}^{\mathrm{T}}, \mathrm{C} \in \mathrm{R}^{\mathrm{N} \times \mathrm{k}}\right\} .
$$

here, each Stiefel manifold $M_{m}=U_{m} \in R^{N \times k}$ : s.t. $U_{m}^{T} U_{m}=I_{K}$.

So, the negative gradient of the objective function can be expressed as:

$$
\mathrm{Z}=-\nabla \operatorname{trace}\left(\mathrm{U}^{\mathrm{T}} \mathrm{LU}\right)=-\mathrm{LU}=\left(\mathrm{Z}_{1}^{\mathrm{T}}, \mathrm{Z}_{2}^{\mathrm{T}}, \cdots, \mathrm{Z}_{\mathrm{m}}^{\mathrm{T}}\right)^{\mathrm{T}}
$$

Then, we search the next point along the direction $\eta_{\mathrm{m}}$ on each tangent vector space of the manifold. Where,

$$
\eta_{\mathrm{m}}=\mathrm{Z}_{\mathrm{m}}-\frac{1}{2} \mathrm{U}^{\mathrm{m}}\left(\left(\mathrm{U}^{\mathrm{m}}\right)^{\mathrm{T}} \mathrm{U}^{\mathrm{m}}+\mathrm{Z}_{\mathrm{m}}^{\mathrm{T}}\right)
$$

$$
\text { Second, we do Backtracking Line Search on Stiefel manifold for problem (9-10). }
$$

The purpose of line search is to find the smallest point of the target function in the search direction. However, it is time-consuming to find the accurate minimum point. The search direction is already approximate, so we just to find the minimum point approximation at a lower cost. Backtracking Line Search (BLS) is such a Line Search algorithm. The idea of the BLS algorithm is to set an initial step size $\alpha_{0}$ in the search direction. Then, if the step size is too large, we reduce the step size until it is appropriate.

Backtracking Line Search in the negative gradient direction of the objective function is as follow:

$$
\mathrm{f}(\mathrm{U}+\alpha \eta) \leq \mathrm{f}(\mathrm{U})+\alpha \mathrm{cm}
$$

$$
\mathrm{m}=\eta^{\mathrm{T}} \mathrm{Z}
$$


175 Where, $\eta$ is the current search direction, $\alpha$ is the step size, and $\mathrm{c}$ is the control parameter,

176 which needs to be manually verified according to the situation.

177 If the current $\mathrm{U}$ does not satisfy inequation (11), then a parameter $\tau$ is required to adjust the step

178 size:

$$
\alpha=\tau \alpha
$$

180 Where, the parameter $\tau$ is controls the reduce search step size.

181 Third, we retract the found points to the manifold with singular value decomposition.

$$
\mathrm{U}=\mathrm{W} \Sigma \mathrm{U}^{\mathrm{T}}, \mathrm{U}=\mathrm{WU}^{\mathrm{T}} .
$$

Algorithm 1: The linear search based on Stiefel manifold

Step 1. The negative gradient direction of the objective function is projected onto Stiefel manifold.

$$
\begin{gathered}
\eta_{\mathrm{m}}=\mathrm{Z}_{\mathrm{m}}-\frac{1}{2} \mathrm{U}^{\mathrm{m}}\left(\left(\mathrm{U}^{\mathrm{m}}\right)^{\mathrm{T}} \mathrm{U}^{\mathrm{m}}+\mathrm{Z}_{\mathrm{m}}^{\mathrm{T}}\right) ; \\
\eta=\left(\eta_{1}^{\mathrm{T}}, \eta_{2}^{\mathrm{T}}, \cdots \eta_{\mathrm{m}}^{\mathrm{T}}\right)^{\mathrm{T}} ;
\end{gathered}
$$

Step 2. Backtracking Linear search in tangent vector space:

$$
U=U+\alpha \eta, \alpha \in(0,1) ;
$$




$$
\begin{gathered}
f(U+\alpha \eta) \leq f(U)+\alpha c m ; \\
m=\eta_{m}{ }^{T} Z
\end{gathered}
$$

Step 3. Retracted the points obtaining in 2 to the Stiefel manifold:

$$
\mathrm{U}=\mathrm{W} \Sigma \mathrm{U}^{\mathrm{T}}, \mathrm{U}=\mathrm{WU}^{\mathrm{T}}
$$

Step 4. Repeat 1- 3 until the convergence condition is satisfied:

$$
\left(\left(r_{-} \text {err_f }>1 e-8\right) \|\left(r_{-} e r r_{-} g>1 e-4\right)\right) \& \&(\text { iters }<1000) \text {; }
$$

Where $r_{-}$err_f represents the relative error of objective function $f, r_{-}$err_grepresents the relative error of the negative gradient of the objective function $\mathrm{Z}$, and iters represents the number of iterations.

Here, we get the solution of the objective function, and then we perform k-means to cluster $U$ and

187 obtain the cluster labels $\mathrm{C}_{1}, \mathrm{C}_{2}, \cdots, \mathrm{C}_{\mathrm{k}}$. Finally, we integrate the clustering results obtained from 188 three omics by using k-nearest neighbor method.

189 Remark: we set $c=6, \tau=0.1$ in our experiment. We will set out the reasons in 3.1.2.

\section{3.Results}

191 In this section, to show the effective of our proposed method, we compare it with AASC algorithm 192 (Huang et al., 2012), SNF method (Wang et al., 2014), MOCMO (Yu et al., 2019), MVSC (Zhang 193 et al., 2015) and Grassmann manifold clustering method (Ding et al., 2018).

\section{$194 \quad$ 3.1The selection of parameter}

\subsubsection{The number of clustering}



did experiments with $\mathrm{k}$ equals 2 to 10 . Then, we choose the number corresponding to the maximum silhouette coefficient. To compare MVSM to other methods, we set $\mathrm{k}$ as a known value.

\subsubsection{The Backtracking Line Search parameters} is the current search direction, $\alpha$ is the step size, and $\mathrm{c}$ is the control parameter, which needs to be manually verified according to the situation. Firstly, we initialize $\alpha=0.01$. During the experiment, it was found that if the value of $\mathrm{c}$ was too small, the step size would not be adjusted during the search process. However, if we want to adjust appropriately, then we need to set the parameter c according to the objective function value and gradient value of the initial point. Therefore, according to several data sets used in the experiment, we set $c=6, \tau=0.1$.

\subsection{Experimental results on simulated datasets}

We refer to the same method in the article (Yu et al., 2019) to verify that this method is suitable for datasets with uneven distribution of underlying clusters. Here we use a simulated dataset.

Since these methods (SNF, AASC and MVSC) were proposed using network tools, we simulate the network structure firstly. For M omics networks, given the number of nodes $\mathrm{N}$, these nodes are assigned to $\mathrm{K}$ clusters. Then, the connections within the same cluster and different clusters are generated. The probability of connections within a given cluster is greater than the probability of connections between clusters. 


$$
\mathrm{P}_{1}=\frac{1}{\mathrm{~N}}\left(\begin{array}{ccc}
16 & 0 & 0 \\
0 & 18 & 0 \\
0 & 0 & 17
\end{array}\right), \quad \mathrm{P}_{2}=\frac{1}{\mathrm{~N}}\left(\begin{array}{ccc}
16 & 0.4 & 0.6 \\
0.4 & 18 & 0.55 \\
0.6 & 0.55 & 17
\end{array}\right)
$$

$$
\mathrm{P}_{3}=\frac{1}{\mathrm{~N}}\left(\begin{array}{ccc}
16 & 0.8 & 1.2 \\
0.8 & 18 & 1.1 \\
1.2 & 1.1 & 17
\end{array}\right), \quad \mathrm{P}_{4}=\frac{1}{\mathrm{~N}}\left(\begin{array}{ccc}
16 & 1.2 & 1.8 \\
1.2 & 18 & 1.65 \\
1.8 & 1.65 & 17
\end{array}\right)
$$

Where, each term $(i, i)$ of the four matrices defines the connection probability within the cluster, and each term $(\mathrm{i}, \mathrm{j}), \mathrm{i} \neq \mathrm{j}$ represents the connection probability between cluster $\mathrm{i}$ and cluster $\mathrm{j}$. For each setting, we consider that in all $\mathrm{M}$ omics, the sample size of each category is the same, and the omics of distribution is different. To see the performance of the method, we tested two settings:

We use the Rand index to evaluate the clustering performance, which is defined as:

with the 'TP', 'TN', 'FP', 'FN' are present the number of the true positive, true negative, false positive, and false negative. 'TP' is defined as the number of intersection nodes in the same cluster, which are also clustered in the same cluster, and other nodes are defined similarly.

Table 1: Performance comparison of different methods. N=150, $\mathrm{M}=3$,

\section{Method}

$$
\mathbf{P}_{1}
$$

$\mathbf{P}_{2}$

$\mathbf{P}_{3}$

$\mathbf{P}_{4}$ 


\begin{tabular}{lcccc}
\hline AASC & 0.73 & 0.73 & 0.73 & 0.73 \\
SNF & 0.68 & 0.67 & 0.67 & 0.67 \\
MVSC & 0.99 & 0.98 & 0.94 & 0.87 \\
MCSM & $\mathbf{1}$ & $\mathbf{0 . 9 9}$ & $\mathbf{0 . 9 4}$ & $\mathbf{0 . 9 7}$ \\
\hline
\end{tabular}

Table 2: Performance comparison of different methods. N=1000, M=6, cluster size:(300,300,400),

\begin{tabular}{ccccc}
\hline Method & $\mathbf{P}_{\mathbf{1}}$ & $\mathbf{P}_{\mathbf{2}}$ & $\mathbf{P}_{\mathbf{3}}$ & $\mathbf{P}_{\mathbf{4}}$ \\
\hline AASC & 0.75 & 0.75 & 0.75 & 0.75 \\
SNF & 0.75 & 0.75 & 0.75 & 0.75 \\
MVSC & 0.93 & 0.93 & 0.93 & 0.93 \\
MCSM & $\mathbf{0 . 9 6}$ & $\mathbf{0 . 9 5}$ & $\mathbf{0 . 9 5}$ & $\mathbf{0 . 9 5}$ \\
\hline
\end{tabular}

2392 , we can see that all four methods cluster nodes with an average Rand index is close to 1 when the 240 cluster sizes of different groups are the same. This also illustrates the importance of data integration.

241 When the size of the underlying cluster is different, because both AASC and SNF set the underlying 242 cluster to be the same, they cannot detect the difference between the different views, so the MVSC 243 and our method have better performance. Table 1 and Table 2 show the mean Rand index and its 
standard deviation in the bracket when the underlying clusters are different for the two setups. By using more strict relaxation of the binary variables, more information of the clusters can be kept.

246 our method out performs MVSC in both setups and when there are less networks, our method 247 performs more stable, which achieves much less standard deviation of the Rand index.

\subsection{Experimental results on real datasets}

In order to prove the effectiveness of our method on the real datasets. We apply our method on

250 multiple datasets analyzed by (Wang et al., 2014), and compare our results with those of SNF fused

251 by similarity network. The final Cox survival P values are listed in Table 3.

Table 3: Comparison of Cox survival p-values.

\begin{tabular}{lcccccc}
\hline Cancer & SNF & Grassmann & MOCMO & AASC & MVSC & MCSM \\
type & \multicolumn{7}{c}{ Cluster } & & & & \\
\hline GBM(3) & 0.0002 & 0.0043 & 0.0019 & 0.0022 & 0.00072 & $\mathbf{0 . 0 0 0 1}$ \\
& & & & & & \\
$\operatorname{BIC}(5)$ & 0.0011 & 0.0002 & $\mathbf{0 . 0 0 0 1 6}$ & 0.00015 & 0.0007 & 0.0025 \\
& & & & & & \\
$\operatorname{SKCM}(4)$ & 0.0001 & 0.19 & 0.00045 & 0.00016 & 0.00045 & $\mathbf{0 . 0 0 0 1}$ \\
& & & & & & \\
$\operatorname{AML}(5)$ & 0.037 & 0.12 & 0.03 & 0.045 & 0.058 & $\mathbf{0 . 0 1 9}$ \\
\hline
\end{tabular}

253 Integrative clustering discovers clinically significant subtypes of cancer. Shown are Kaplan Meier

254 plots of the overall survival of integrative clusters for Glioblastoma Multiforme (GBM) (a), breast

255 Invasive carcinoma (BIC) (b), Skim Cutaneous Melanoma (SKCM) (c) and Acute Myeloid

256 Leukemia (AML) (d) in figure 2. P-values are computed from the log rank test. 


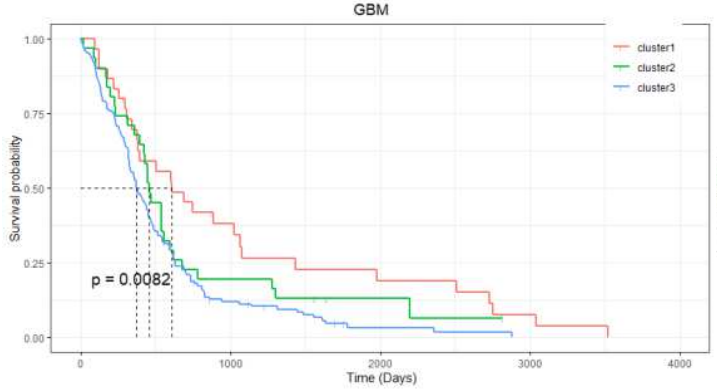

(a)

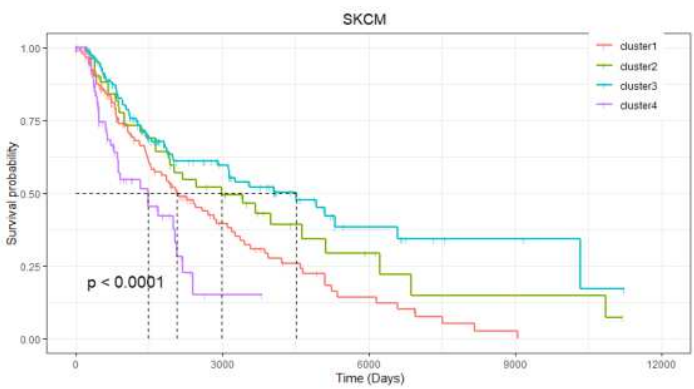

(c)

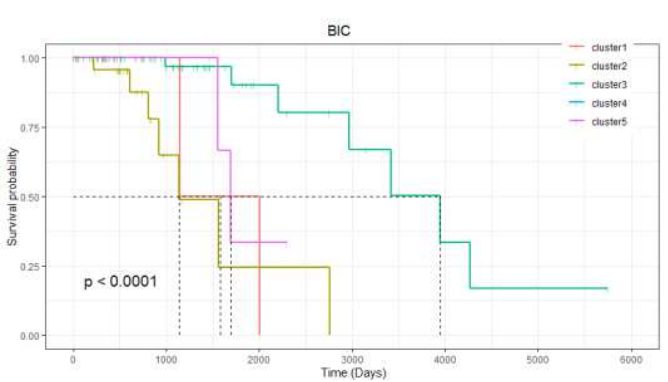

(b)

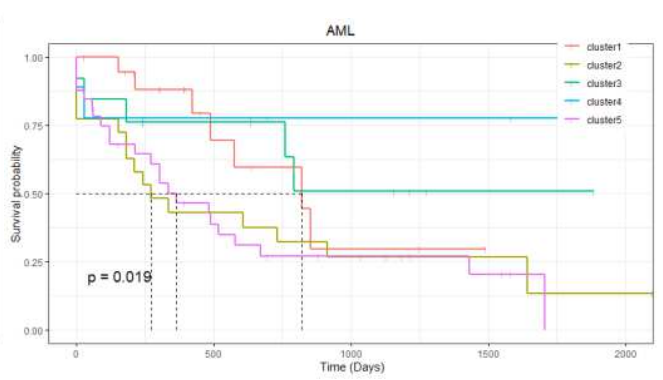

(d)

Figure 2: Survival plots for GBM, BIC, SKCM, and AML tumors.

It can be seen from the Table 3, in three of the four cancers datasets (GBM, BIC, SKCM and AML), our method obtains more significant differences than comparison methods in survival time.

For BIC dataset, the insignificant difference may be due to the small cluster difference of the data itself. Survival plots for GBM, BIC, SKCM, and AML tumors are shown in Figure 2. Our proposed method can also be extended to improve the survival rate prediction task.

\subsection{A case study: Comparison of clusterings to established subtypes}

In order to compare the results of our clustering with the established biological subtypes, we downloaded the clinical data of 215 GBMs from the cBio Cancer Genomis Portal 
271 exist four established subtypes determined by patients' gene expression profiles, which are Classical,

272 Mesenchymal, Neural and Proneural (Verhaak et al., 2010), and a subtype called Glioma-CpG island

273 methylator phenotype (G-CIMP) generated by DNA methylation clustering (Noushmehr et al.,

274 2010). Comparison of our GBM clustering with these existing subtypes (Table 4) shows that our

275 method not only reflects evidence from one data type, but also finds a cluster that considers both

276 gene expression and DNA methylation information.

Table 4: Comparison of clusterings to established subtypes

\begin{tabular}{|c|c|c|c|c|c|c|}
\hline \multirow[b]{2}{*}{ Clusters } & \multicolumn{4}{|c|}{ Gene expression subtypes } & \multicolumn{2}{|c|}{$\begin{array}{l}\text { DNA methylation subtypes } \\
\text { (Noushmehr et al., 2010) }\end{array}$} \\
\hline & Classical & Mesenchymal & Neural & Proneural & G-CIMP & Non-G-CIMP \\
\hline Cluster1 & 0 & 0 & 1 & 13 & 16 & 14 \\
\hline Cluster2 & 2 & 25 & 2 & 2 & 0 & 31 \\
\hline Cluster3 & 55 & 41 & 31 & 24 & 2 & 152 \\
\hline
\end{tabular}

For gene expression subtypes, it can be seen that cluster 1 mainly contains Proneural subtype,

280 cluster 2 mainly contains Proneural subtype, and they have strong enrichment. However, for DNA

281 methylation subtypes, G-CIMP subtypes are mainly distributed in cluster 1. If only the DNA methylation information is considered, cluster2 and cluster3 are likely to merge. So, we can conclude that it's important to consider both gene expression and DNA methylation information. 

to temozolomide (TMZ) treatment of the GBMs. TMZ is an alkylation agent that causes incorrect pairing of thymine during DNA replication. In the GBM dataset, 105 patients were treated with TMZ. Figure 3 indicated that the TMZ-treated samples had different drug responses compared to the samples not treated with the drug. For different clusters, the degree of drug response of TMZ was also different. Compared with Cluster 1 and Cluster 2, patients in Cluster 3 had significantly increased survival time after treatment with TMZ (P value using Cox log-rank test $=0.0001$ ), and this medication was also more meaningful. The results show that the clusters we obtained can be used as a reference for identifying the effectiveness of drugs.

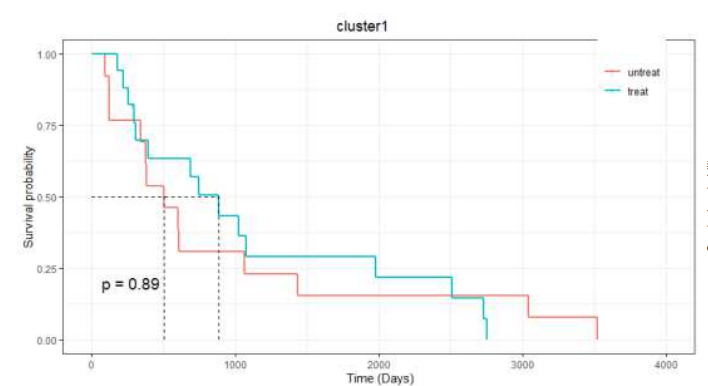

(a)

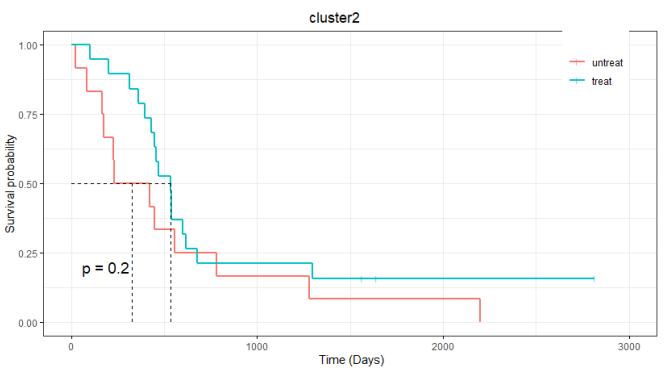

(b)

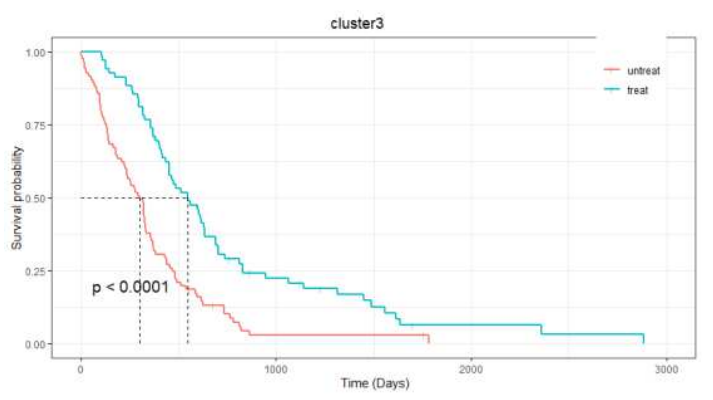

(c)

Figure 3: Survival analysis of GBM patients for treatment with Temozolomide in the different clusterings. 

the underlying clusters are consistent, and as we reviewed in the first section, several approaches have been proposed. When the underlying cluster is different, some methods are proposed to find different clusters. However, as we know, both consistent and differentiated clusters can exist at the same time. This leads us to study multi-view simultaneous clustering to find both consistent and different cluster data. In this paper, we propose a multi-view clustering model. On the basis of manifold optimization, the algorithm for formula optimization is proposed. Simulation results show that the performance of the proposed method is better than that of the existing algorithm under the same underlying cluster condition. We download the gene expression, miRNA expression and DNA methylation datasets of GBM, BIC, SKCM and AML from TCGA, and also carry out numerical experiments, showing that our method is superior to several comparison methods. In the future work, the cluster difference problem is still worth researching, and we will integrate other omics information such as gene mutation data.

\section{Declarations}

Ethics approval and consent to participate: Not applicable.

315 Consent for publication: Not applicable.

\section{Availability of data and materials:}

317 All data generated or analysed during this study are included in this published article [and its suppl ementary information files]

Competing interests: Not applicable.

Funding: Not applicable. 
manifold method (MCSM) and the numerical simulation results of MCSM model. Z JP' contribution lies in the embellishment of the article. Z CH' contribution lies in the thought guidance of the method.

Acknowledgements: This work was supported by grants from the Xinjiang Autonomous Region

Foundation of China (No. U19A2064, 61873001).

\section{References}

[1] Absil PA, Mahony R and Sepulchre R. Optimization Algorithms on Matrix Manifolds. Princeton University Press, Princeton, NJ (2008).11462-11467. doi: 10.1515/9781400830244

[2] Alon U, Barkai N, Notterman DA, Gish K, Ybarra S, Mack D, and Levine AJ. Broad patterns of gene expression revealed by clustering analysis of tumor and normal colon tissues probed

[4] Bendor A, Shamir R and Yakhini Z. Clustering gene expression patterns. Journal of

[5] Bickel PJ and Chen A. A nonparametric view of network models and new managirvan and by oligonucleotide arrays. Proceedings of the National Academy of Sciences of the United States of America (1999) 96(12): 6745-6750. doi: 10.1073 / pnas.96.12.6745

[3] Ashburner M, Ball CA, Botstein D and Blake JA. Gene ontology: tool for the unification of biology. Nature Genetics (2000) 25(1): 25-29. doi: 10.1038/75556

$$
\text { Computational Biology (1999) 6: 281-297. doi: 10.1145/299432.299448 }
$$
other modularities. Proceedings of the National Academy of Sciences of the United States of America (2009)106(50): 21068-21073. doi: 10.1073/pnas.0907096106 
[6] Cancer Genome Atlas Research Network. Comprehensive genomic characterization of squamous cell lung cancers. Nature (2012) 489: 519-525. doi: 10.1016/j.ypat.2012.11.048

[7] Chang HY, Nuyten DSA, Sneddon JB, Hastie Tibshirani R, Dai HY and He YD. Robustness, scalability and integration of a wound-response gene expression signature in predicting breast cancer survival. Proceedings of the National Academy of Sciences of the United States of America (2005) 102(10): 3738-3743. doi:10.1073/pnas.0409462102

[8] Chalise P, Koestler DC, Bimall M, Yu Q. Integrative clustering methods for high dimensional molecular data. Translational cancer research (2014) 3(3), 202-216. doi: 10.3978/j.issn.2218676X.2014.06.03

[9] Chen C, Ng MK, and Zhang S. Block spectral clustering methods for multiple graphs. Numerical Linear Algebra with Applications (2017) 24(1): 1-20. doi: 10.1002/nla.2075

[10] Dai LY, Feng CM, Liu JX, Zheng CH, Yu J. Robust graph regularized discriminative nonnegative matrix factorization for characteristic gene selection. IEEE International Conference on Bioinformatics and Biomedicine (2016) 1253-1258. doi: 10.1109/BIBM.2016.7822698

[11] Ding H, Michael S, Wang C. Integrative cancer patient stratication via subspace merging. Bioinformatics (2018) 35(10): 1653-1659. doi: 10.1093/bioinformatics/bty866

[12] Fridley BL, Lund SP, Jenkins GD, Wang LW. A Bayesian integrative genomic model for pathway analysis of complex traits. Genetic epidemiology (2012) 36:352-359. doi: 10.1002/gepi.21628 
[13] Ge SG, Xia J, Sha W, Zheng CH. Cancer subtype discovery based on integrative model of multigenomic data. IEEE/ACM transactions on computational biology and bioinformatics (2017) 14(5): 1115-1121. doi: 10.1109/TCBB.2016.2621769

[14] Ha MJ, Baladandayuthapani V and Do KA. Dingo: Differential network analysis in genomics. Bioinformatics (2015) 31(21): 3413-3420. doi: 10.1093/bioinformatics/btv406

[15] Noushmehr H, Weisenberger DJ, Diefes K, Phillips HS, Pujara K, Berman BP and Cancer Genome Atlas Research Network. Identification of a $\mathrm{CpG}$ island methylator phenotype that defines a distinct subgroup of glioma. Cancer cell (2010) 17(5): 510-522.

[16] Huang HC, Chuang YY and Chen CS. Affinity aggregation for spectral clustering. Conference on Computer Vision and Pattern Recognition (2012) 2012: 773-780. doi: 10.1109/CVPR.2012.6247748

[17] Huang BY, Zhang HB, Gu LJ, Ye BX and Xiong X. Advances in immunotherapy for glioblastoma multiforme. Clinical Developmental Immunology (2017) 2017(3): 1-11. doi: $10.1155 / 2017 / 3597613$

[18] Huang E, Cheng SH, Dressman H and Pittman J. Gene expression predictors of breast cancer outcomes. The Lancet (2003) 361(9369): 1590-1596. doi: 10.1016/S0140-6736(03)13308-9

[19] Kanehisa M, Goto S, Furumichi M, Tanabe M and Hirakawa M. Kegg for representation and analysis of molecular networks involving diseases and drugs. Nucleic Acids Research (2010) 38(suppl_1): D355-D360. doi: 10.1142/9781848165632_0020

[20] Kumar R, Kamdar D, Madden L, Hills C. Th1/th2 cytokine imbalance in meningioma, 
anaplastic astrocytoma and glioblastoma multiforme patients. Oncology Reports (2006) 15(6): 1513-1516. doi: 10.3892/or.15.6.1513

384

[21] Shen R, Olshen AB and Ladanyi M. Integrative clustering of multiple genomic data types using a joint latent variable model with application to breast and lung cancer subtype analysis. Bioinformatics (2010) 25(22): 2906-2912. doi: 10.1093/bioinformatics/btp659

[22] Shen R, Mo Q, Schultz N and Seshan VE. Integrative subtype discovery in glioblastoma using icluster. Plosone (2012) 7(4): e35236. doi: 10.1371/journal.pone.0035236

[23] Speicher NK and Pfeifer N. Integrating different data types by regularized unsupervised multiple kernel learning with application to cancer subtype discovery Bioinformatics (2015) 31(12): i268-i275. doi: 10.1093/bioinformatics/btv244

[24] Stefansson OA, Moran S, Gomez A and Sayols S. A DNA methylation-based definition of biologically distinct breast cancer subtypes. Molecular Oncology (2015) 9(3): 555-568. doi: 10.1016/j.molonc.2014.10.012

[25] Verhaak RGW, Hoadley KA, Purdom E, Wang V, Qi Y, and Wilkerson MD. Integrated genomic analysis identifies clinically relevant subtypes of glioblastoma characterized by abnormalities in pdgfra, idh1, egfr, and nf1. Cancer Cell (2010) 17(1): 98-110.

[26] West M, Blanchette C, Dressman H and Huang E. Predicting the clinical status of human breast cancer by using gene expression profiles. Proceedings of the National Academy of Sciences of the United States of America (2001) 98(20): 11462-11467. doi: 10.1073/pnas.201162998

[27] Wang B, Mezlini AM, Demir F and Fiume M. Similarity network fusion for aggregating data 
403

404

405

406

407

408

409

410

411

412

413

414

415

416

417

[28] Yuan Y, Savage RS, Markowetz F. Patient-specific data fusion defines prognostic cancer

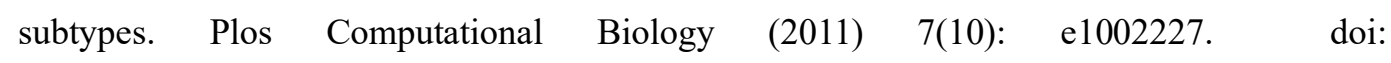
10.1371/journal.pcbi.1002227

[29] Yu Y, Zhang LH and Zhang SQ. Simultaneous clusterin of multiview biomedical data using manifold optimization, Bioinformatics (2019) 35(20): 4029-4037. doi: 10.1093/bioinformatics/btz217

[30] Zhang D, Chen P, Zheng CH, and Xia JF. Identification of ovarian cancer subtype-specific network modules and candidate drivers through an integrative genomics approach. Oncotarget (2016) 7(4): 4298. doi: 10.18632/oncotarget.6774

[31] Zhang S, Zhao H and Ng MK. Functional module analysis for gene coexpression networks with network integration. IEEE/ACM Transactions on Computational Biology and Bioinformatics (2015) 12(5): 1146-1160. doi: 10.1109/TCBB.2015.2396073

[32] Zheng CH, Ng TY, Zhang L, Shiu, C. K., \& Wang, H. Q. Tumor classification based on nonnegative matrix factorization using gene expression data. IEEE transactions on nanobioscience (2011) 10(2): 86-93. doi: 10.1109/TNB.2011.2144998

[33] Zheng CH, Yang W, Chong YW, Xia JF. Identification of mutated driver pathways in cancer using a multi-objective optimization model. Computers in Biology and Medicine (2016) 72: 22-29. doi: 10.1016/j.compbiomed.2016.03.002 
Figures
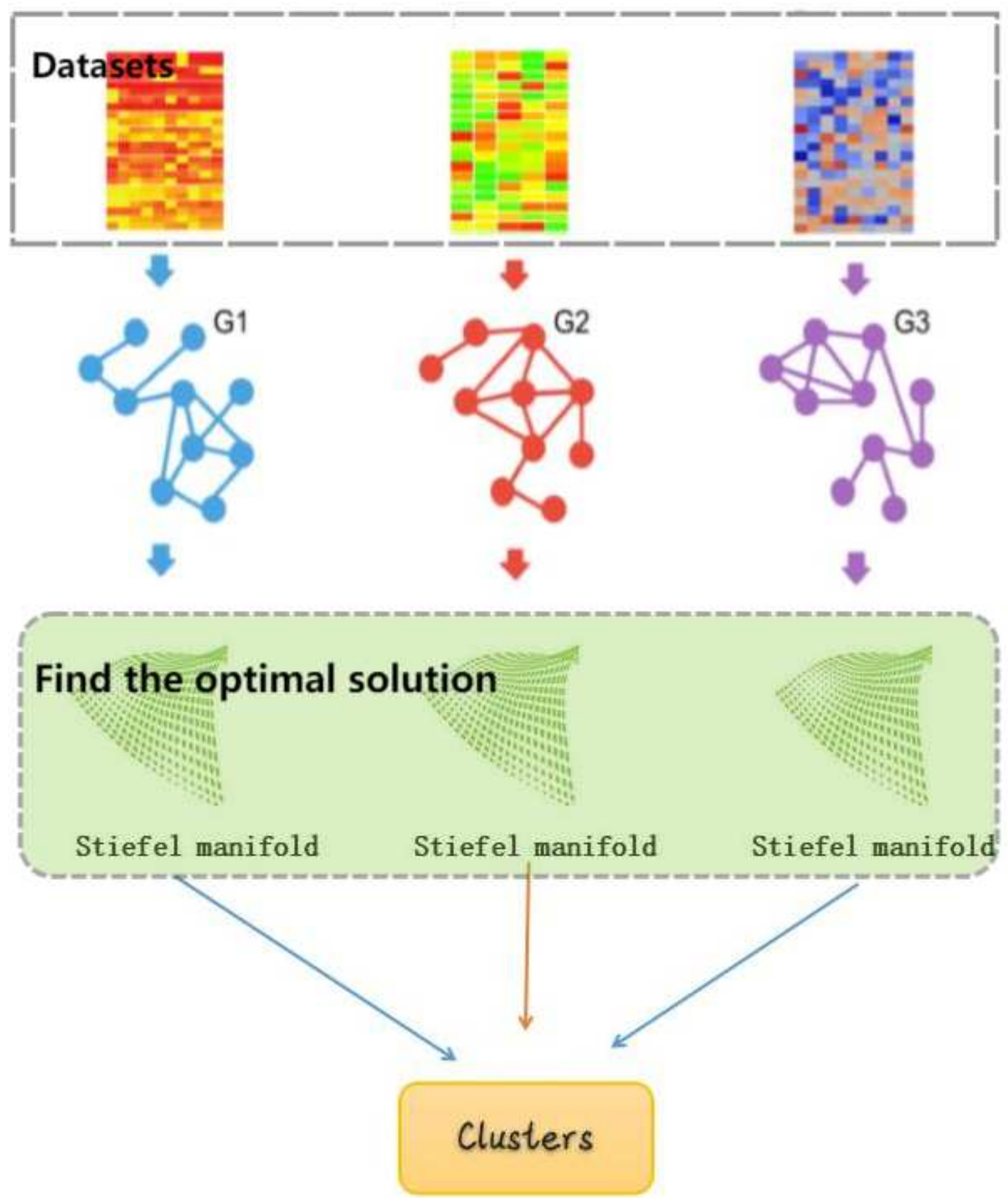

Figure 1

The process of MCSM method. 


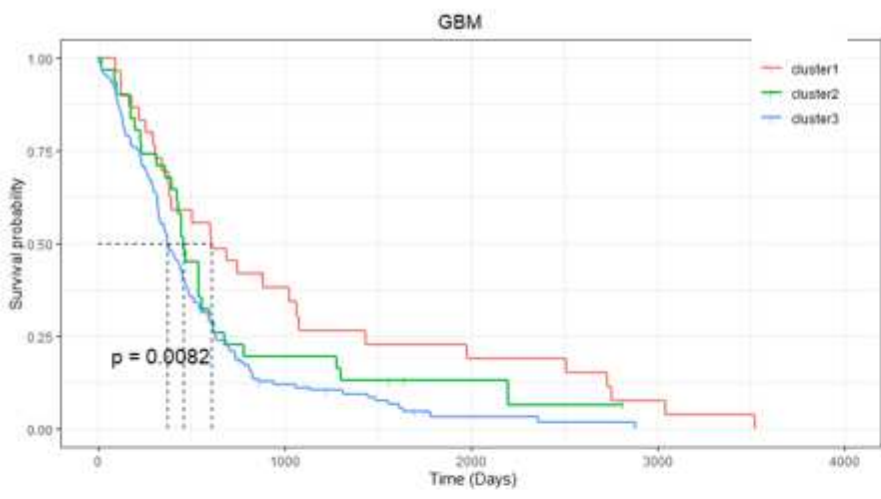

(a)

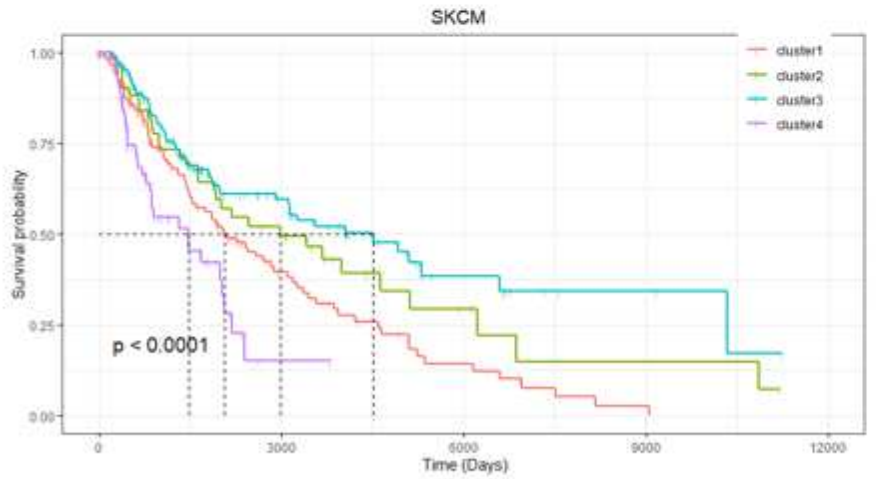

(c)

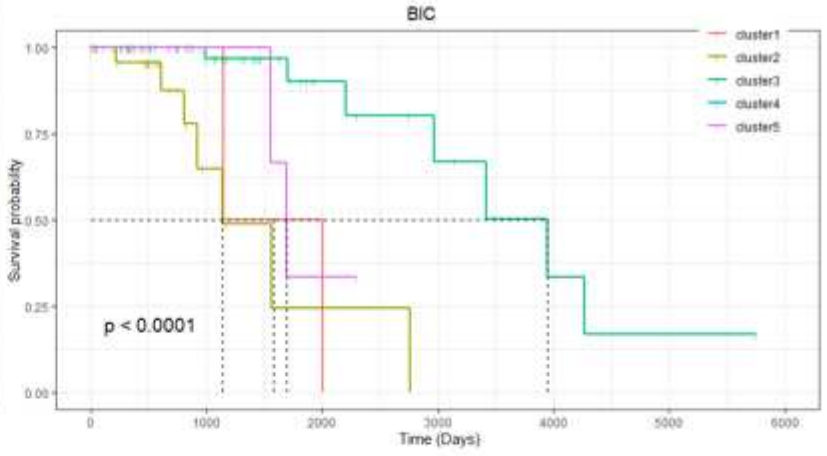

(b)

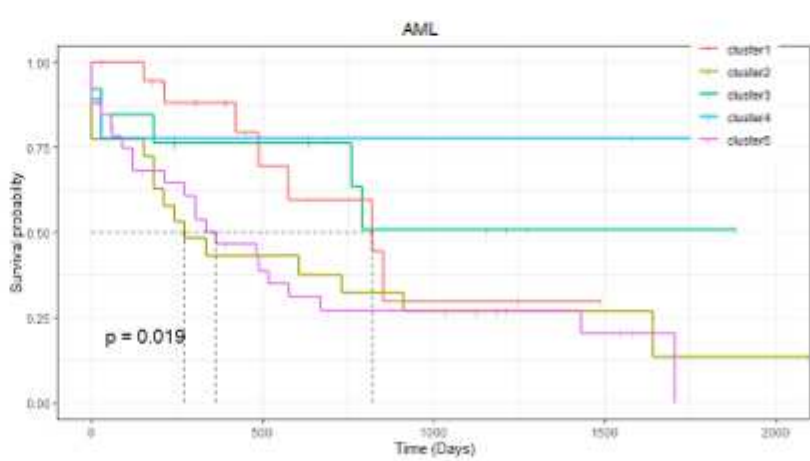

(d)

Figure 2

Survival plots for GBM, BIC, SKCM, and AML tumors. 


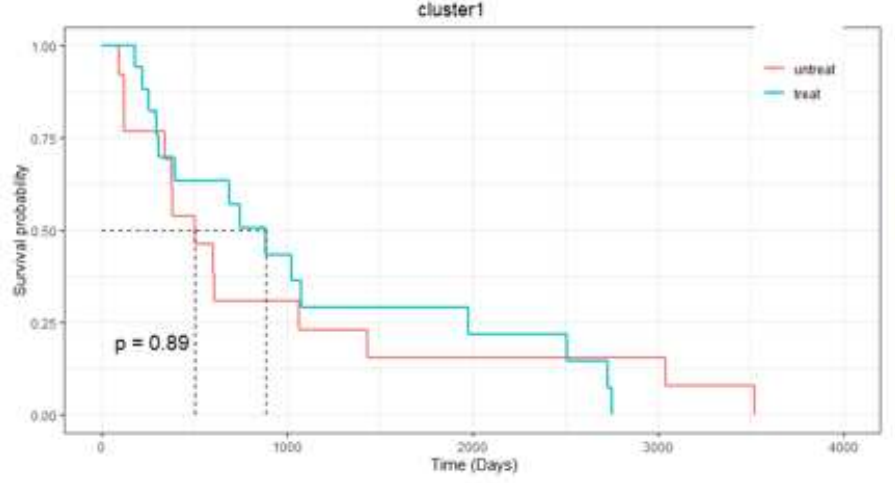

(a)

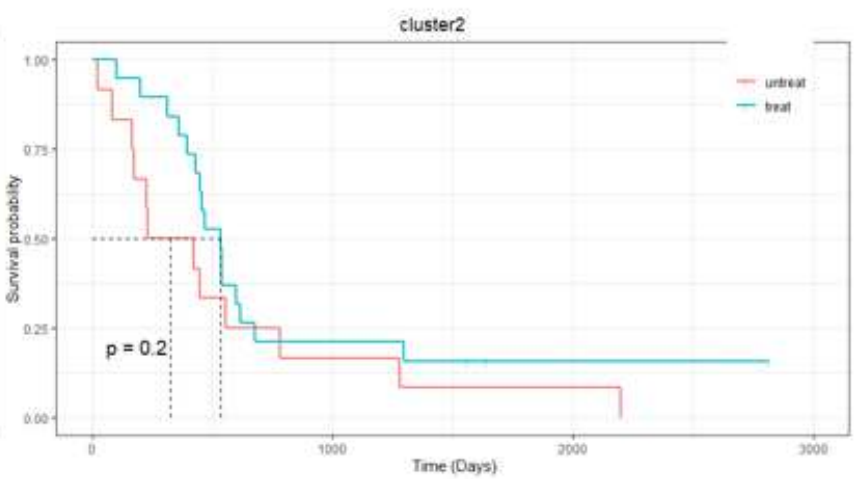

(b)

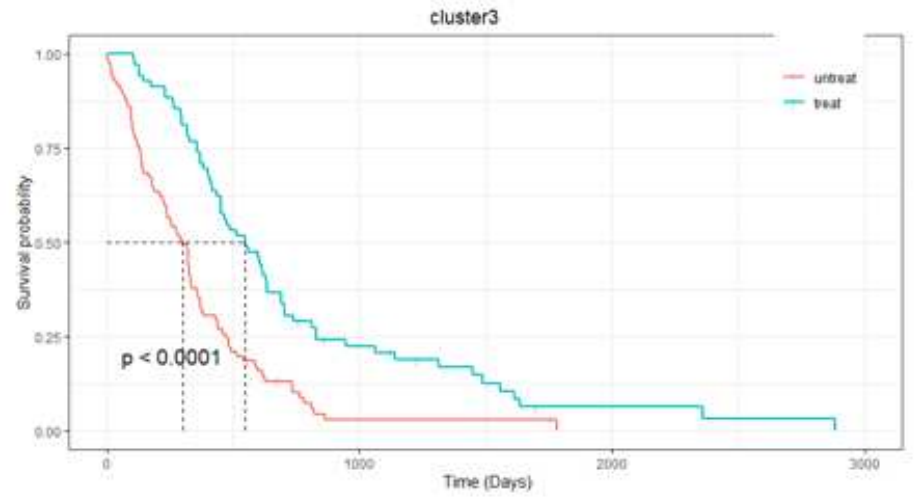

(c)

\section{Figure 3}

Survival analysis of GBM patients for treatment with Temozolomide in the different clusterings. 